\title{
What stops hospital clinical staff from following protocols? An analysis of the incidence and factors behind the failure of bedside clinical staff to activate the rapid response system in a multi-campus Australian metropolitan healthcare service
}

\author{
Bill Shearer, ${ }^{1,2}$ Stuart Marshall, ${ }^{2,3}$ Michael David Buist, ${ }^{4}$ Monica Finnigan, ${ }^{1}$ \\ Simon Kitto, ${ }^{5}$ Tonina Hore, ${ }^{6}$ Tamica Sturgess, ${ }^{6}$ Stuart Wilson, ${ }^{6}$ Wayne Ramsay ${ }^{6}$
}

- An additional appendix is published online only. To view this file please visit the journal online (http:// qualitysafety.bmj.com/ content/21/7.toc).

For numbered affiliations see end of article.

Correspondence to Professor Michael David Buist, School of Medicine, University of Tasmania, Private Bag 3513, Burnie, TAS 7320, Australia; michael.buist@utas.edu.au

Guarantor for the study: Dr Bill Shearer.

Accepted 13 March 2012 Published Online First 23 May 2012

\section{ABSTRACT}

Objective: To explore the causes of failure to activate the rapid response system (RRS). The organisation has a recognised incidence of staff failing to act when confronted with a deteriorating patient and leading to adverse outcomes.

Design: A multi-method study using the following: a point prevalence survey to determine the incidence of abnormal simple bedside observations and activation of the rapid response team by clinical staff; a prospective audit of all patients experiencing a cardiac arrest, unplanned intensive care unit admission or death over an 8-week period; structured interviews of staff to explore cognitive and sociocultural barriers to activating the RRS.

Setting: Southern Health is a comprehensive healthcare network with 570 adult in-patient beds across four metropolitan teaching hospitals in the south-eastern sector of Melbourne.

Measurements: Frequency of physiological instability and outcomes within the in-patient hospital population. Qualitative data from staff interviews were thematically coded.

Results: The incidence of physiological instability in the acute adult population was $4.04 \%$. Nearly half of these patients (42\%) did not receive an appropriate clinical response from the staff, despite most (69.2\%) recognising their patient met physiological criteria for activating the RRS, and being 'quite', or 'very' concerned about their patient (75.8\%). Structured interviews with 91 staff members identified predominantly sociocultural reasons for failure to activate the RRS.

Conclusions: Despite an organisational commitment to the RRS, clinical staff act on local cultural rules within the clinical environment that are usually not explicit.
Better understanding of these informal rules may lead to more appropriate activation of the RRS.

\section{OBJECTIVE}

Failure of bedside hospital clinical staff to follow established treatment protocols has been identified as a common factor in patients having an adverse event during their hospitalisation. Post hoc analysis ${ }^{1}$ of the data from the Australian Quality and Health Care Study (AQHCS) ${ }^{2}$ found that 'that misapplication of, or failure to apply a rule; or use of a bad or inadequate rule' and 'violation of a protocol or rule' together occurred in $13.6 \%$ of the adverse events identified in the AQHCS. These two iterative categories of adverse event causation were associated with death or permanent disability in $26 \%$ and $28 \%$ respectively of adverse events identified in the AQHCS. Of most concern, the authors rated the degree of preventability of these two causation categories at $90 \%$ and $80 \%$ respectively based on the actual adverse events in the AQHCS. Likewise, a number of smaller mostly retrospective single institution studies have found various associations between failure to follow established treatment protocols and guidelines for clinically deteriorating patients and increased mortality. ${ }^{3-7}$

One strategy to counter the problem of inhospital adverse events has been the use of the 
rapid response system (RRS) to manage clinically deteriorating patients. In essence a RRS has two essential components: first an afferent arm that is a system of rules that determine the criteria for activation of the rapid response team; and second, the efferent arm which is the system of response once the activation criteria are breached. Varying types of RRS are now common in many European, North American and Australasian hospitals. ${ }^{8} 9$ In its simplest form a RRS is merely a rule or protocol that requires the bedside clinical staff to comply with the critical requirement of activating the RRS for it to be of benefit. ${ }^{10}$ In the Medical Emergency Response Intervention Trial (MERIT).${ }^{11}$ there was a significant rate of failure to activate such a system in the control and intervention hospitals that may have contributed to the equivocal results of the study. Specifically, in the 11 control hospitals over the 6-month study there were 246 cardiac arrests. In $44 \%$ of these arrests the bedside staff did not activate an emergency response despite the observations fulfilling the response criteria for an emergency team call. Similarly, of the 12 hospitals assigned to the RRS intervention, there was still a failure rate of $30 \%$ to activate the RRS in the 250 cardiac arrests that occurred.

Anecdotally, failure to activate the RRS has been thought to be associated with inadequate education about recognition of clinically deteriorating patients or insufficient knowledge of the RRS. In support of this is a post hoc analysis of the MERIT data ${ }^{12}$ that indicates an inverse relationship between the frequency of emergency response team calls (in the control and intervention hospitals) and the primary outcome measures of cardiac arrest, critical unit admission and hospital mortality. Similarly, Santamaria $e a^{13}{ }^{13}$ reported data from one of the intervention hospitals of the MERIT study 5 years after the study suggesting that an intervention like a RRS takes years not months to derive benefits. Theoretically then, the rate of RRS activation should be high in a system where it has been in place for many years and the clinical staff have adequate training.

In 2007 the Quality and Safety Unit, Southern Health, Melbourne identified 11 sentinel cases in which significant patient harm occurred in association with the failure of bedside staff to call the medical emergency team. This was despite the fact that our organisation had a mature policy and procedure in place for the activation of the RRS that has been well established with an organisational commitment to its use. ${ }^{14}{ }^{15}$ Attempts to rationalise and pattern-match the underlying features of these incidents using clinical reviews and root cause analyses were unsuccessful. There appeared to be no obvious cause in terms of poor staff training, lack of motivation or understanding of the patients' clinical states. ${ }^{16}$

While previous studies ${ }^{17-21}$ have documented the incidence of failure of bedside clinical staff to activate an emergency response team, to our knowledge no study has investigated the reasons why staff do not activate the RRS. As such, we undertook a multi-method examination to determine the incidence of clinical staff failing to call the RRS and the human and sociological factors that may be involved.

\section{DESIGN}

\section{Ethics approval}

The Southern Health Research and Ethics Committee exempted this study from requiring ethics approval because it fulfilled the NHMRC criteria for a quality assurance project.

\section{Methods and participants}

We have previously published the methodology for this study. ${ }^{22}$ In summary there were three parts to this study.

\section{Point prevalence study of missed RRS incidence}

On 17 April 2009 between 11:00 and 12:00 all adult inpatient observation charts were reviewed (excluding critical care areas such as intensive care units (ICUs), high dependency units, the emergency department and the theatre complex). Evidence of documented physiological instability as defined by the RRS activation criteria (table 1) was determined by review of the patient observation charts over the previous $24 \mathrm{~h}$. If patients fulfilled these physiological criteria then the actions of the staff in response to clinical instability were recorded. If the RRS was not activated when RRS activation criteria were met, the event was termed a 'missed RRS call'.

\section{Prospective study of missed RRS calls}

Over an 8-week period from 26 April to 7 July 2009 all cardiac arrests and unplanned ICU admissions from the ward areas were examined to determine if the patient fulfilled the activation criteria for a RRS call (table 1) for

Table 1 The rapid response system physiological criteria or triggers

\begin{tabular}{|c|c|}
\hline Airway & $\begin{array}{l}\text { Respiratory distress } \\
\text { Threatened airway }\end{array}$ \\
\hline Breathing & $\begin{array}{l}\text { Respiratory rate }>30 \text { breaths per min } \\
\text { Respiratory rate }<6 \text { breaths per min } \\
\text { Oxygen saturation }<90 \% \text { on oxygen } \\
\text { Difficulty speaking }\end{array}$ \\
\hline Circulation & $\begin{array}{l}\text { Blood pressure }<90 \mathrm{~mm} \mathrm{Hg} \text { despite } \\
\text { treatment } \\
\text { Pulse rate }>130 \text { beats per min }\end{array}$ \\
\hline Neurology & $\begin{array}{l}\text { Decreased level of consciousness } \\
\text { Fitting }\end{array}$ \\
\hline Other & $\begin{array}{l}\text { Concerned } \\
\text { Need for treatment and prompt help }\end{array}$ \\
\hline
\end{tabular}


a minimum of $4 \mathrm{~h}$ in the $24 \mathrm{~h}$ period prior to the index event. In the event that the RRS was not activated, all the clinical staff involved were interviewed (see below) and these events were also labelled 'missed RRS call'.

\section{Interviews of staff members involved in missed RRS calls}

The clinical staff involved in 'missed RRS calls' in the first two parts of the study were interviewed (online appendix 1). These staff members included the junior medical and nursing staff and members of the medical emergency or intensive care teams that were called to assist. The interview questions were derived from the cognitive theory of situation awareness ${ }^{23}$ and from brief open-ended questions designed to explore the sociocultural barriers identified in health systems. ${ }^{24}$

Quantitative data were recorded as frequency data as this was an exploratory study and not designed to distinguish adverse event rates among clinical areas. Interview responses were coded independently by two coders for themes and the coding system iteratively developed until agreement was achieved. ${ }^{25}$

\section{Setting}

Southern Health is a comprehensive university tertiary healthcare provider for the south-eastern suburbs of Melbourne. Over 13000 staff service a catchment population of 880000 and provide acute health services at four hospital sites; all acute services are provided with the exception of burns, spinal injury and major organ transplantation. Southern Health was an early and enthusiastic adopter of the RRS with establishment of the medical emergency team in one hospital in 1996. This resulted in substantial and significant improvements in patient outcome $^{14} 15$ and this system of care was standardised throughout the organisation by 2006 . Additionally our organisation committed to training all junior members of clinical staff in the purpose built simulation centre. ${ }^{26}$

\section{RESULTS}

\section{Incidence of physiological and missed RRS calls}

On the day of the point prevalence study, $23(4.04 \%)$ patients fulfilled the activation criteria for a RRS call in the $24 \mathrm{~h}$ before the index event according to the 570 observation charts that were surveyed (table 2). The RRS was not activated in $10(1.75 \%)$ of these patients (missed RRS call). The research team activated the RRS for one patient who was subsequently admitted to the ICU and underwent emergency surgery that day. Hypotension and oxygen de-saturation were the most common reasons for RRS call activation ( $73.3 \%$ and $80.0 \%$ respectively).

\section{Prospective identification of missed RRS calls}

Over the prospective study period a further 31 cases were identified across the four acute hospital sites (table 2).

Interviews of clinical staff involved in a missed RRS call Incomplete qualitative data were obtained from 8 of the 91 interviews for questions relating to sociocultural barriers to activation of the RRS and so were not used.

Table 2 Adverse event and rapid response system (RRS) data collected from the point prevalence study and the prospective study period

\begin{tabular}{|c|c|c|c|c|c|}
\hline & \multicolumn{5}{|c|}{ Frequency (per 1000 bed days) } \\
\hline \multicolumn{6}{|l|}{ Point prevalence study } \\
\hline Acute adult patients & 59 & 287 & 177 & 47 & 570 \\
\hline $\begin{array}{l}\text { Patients who } \\
\text { met RRS activation criteria }\end{array}$ & 0 & 13 & 8 & 2 & $23(4.04 \%)$ \\
\hline Missed RRS calls & 0 & 5 & 3 & 2 & $10(1.75 \%)$ \\
\hline \multicolumn{6}{|l|}{ Prospective study } \\
\hline Bed days & 2643 & 13719 (8 weeks) & 16756 (10 weeks) & 3642 & 36760 \\
\hline RRS activation & $14(5.30)$ & $142(10.4)$ & $180(10.7)$ & $21(5.67)$ & $357(9.71)$ \\
\hline Cardiac arrest calls & $2(0.76)$ & $36(2.62)$ & $15(0.90)$ & $1(0.27)$ & $54(1.47)$ \\
\hline Unplanned ICU admission & 0 & $15(1.09)$ & $8(0.48)$ & $2(0.55)$ & $25(0.68)$ \\
\hline $\begin{array}{l}\text { ICU admission following } \\
\text { RRS/cardiac arrest call }\end{array}$ & $1(0.38)$ & $69(5.03)$ & $52(3.10)$ & $5(1.37)$ & $127(3.45)$ \\
\hline Death & 0 & $4(0.29)$ & $3(0.18)$ & 0 & $7(0.19)$ \\
\hline $\begin{array}{l}\text { Missed RRS calls-late } \\
\text { RRS calls/ICU admissions/deaths }\end{array}$ & 0 & $20(1.46)$ & $10(0.60)$ & $1(0.27)$ & $31(0.84)$ \\
\hline Interviews from missed RRS calls & 0 & $\begin{array}{l}58 \text { (26 nurses, } \\
18 \text { medical staff, } \\
14 \text { ICU nursing } \\
\text { and medical staff) }\end{array}$ & $\begin{array}{l}32 \text { (17 nurses, } \\
11 \text { medical staff, } \\
4 \text { ICU nursing } \\
\text { and medical staff) }\end{array}$ & $\begin{array}{l}1 \text { (junior } \\
\text { medical } \\
\text { staff) }\end{array}$ & $\begin{array}{l}91 \text { (qualitative } \\
\text { data available } \\
\text { from } 83 \text { ) }\end{array}$ \\
\hline
\end{tabular}


Cognitive barriers for failing to activate the RRS

Even though they did not activate the RRS, the majority of staff in the prospective study (63 of 91, 69\%) recognised that the patients met the calling criteria. Furthermore, 69 of the 91 respondents $(75.8 \%)$ were either 'quite concerned' (33 of 91, 36.3\%) or 'very concerned' (36 of 91, 39.6\%) about their patient at this time. Fifty-seven respondents $(62 \%)$ were unaware that their patient had been classified as a missed RRS, and of these, $27(47.4 \%)$ thought the patient would deteriorate despite treatment.

\section{Sociocultural barriers of failure to activate RRS}

Ten themes were identified in the responses of staff involved with missed RRS calls. The staff believed they would be perceived by their nursing and medical colleagues in similar ways had they activated the RRS. Many stated their colleagues would be supportive of whatever actions they felt necessary (40 of $83,48.2 \%$ for nursing colleagues, 21 of $83,25.3 \%$ for medical colleagues) with fewer concerned there would be a negative or hostile response (11 of 83, 13.3\% for nursing colleagues and 22 of $83,26.5 \%$ for medical colleagues) (figure 1 ).

One of the main barriers given by respondents was a feeling that they should be able to manage patients by themselves on the ward (34 of 83, $41.0 \%$ for nursing staff and 33 of $83,39.8 \%$ for medical staff).

\section{Actions and explanations for the delay of activation of the RRS}

When activation of the RRS was delayed four themes were identified, including the need to wait for further investigations, and treatment and reviews by the treating and ICU teams. (table 3). When the RRS was not activated at all a further eight themes were identified (table 3). The most common of these were that the RRS did not need to be activated and that the assembled team had the necessary expertise. This was most commonly noted when the

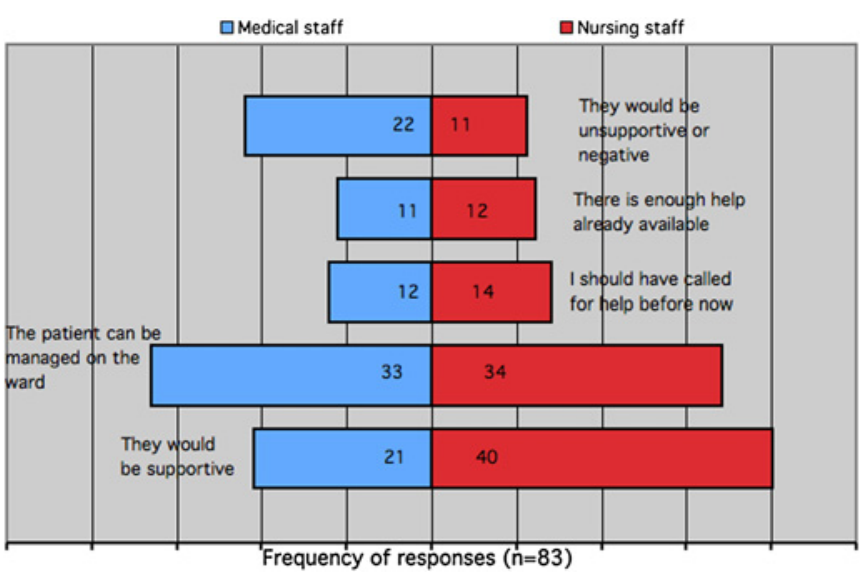

Figure 1 Thematic analysis of responses to the question: 'How would you think you would be perceived by your nursing/ medical colleagues if you called a MET call now?' physiological instability was in the area of expertise of the treating team (eg, tachycardia on the cardiology ward). A significant proportion of interviewees (25 of 83, 30.1\%) believed the patient had already been discussed with the ICU team and felt that continued responsibility for the patient was unclear.

\section{DISCUSSION}

\section{Principle findings}

On the day of the point prevalence study, 1 in 25 patients across the four acute care hospitals fulfilled the activation criteria for the RRS. The RRS was not activated in $42 \%$ of instances, despite the maturity of the RRS and educational support for its use in our organisation.

Interviews with staff involved with missed RRS calls revealed that, even though they did not activate the RRS, nearly three-quarters of respondents recognised that their patients met the RRS activation criteria and a similar proportion were concerned about their patients' wellbeing. In these situations the bedside staff took other actions to manage their patients, which in most cases involved further investigations, treatments or clinical reviews (table 3). Of concern though, a minority of respondents (nurses 13\% and doctors 26\%) were concerned about a negative or hostile reaction from colleagues for activating the RRS.

The most common reason for failure to activate the RRS was that the bedside staff felt that the clinical situation was under control in the ward setting. The second most common reason was that the critical care team had already been involved but that there were no critical care beds available. Less common reasons were a feeling that the bedside clinical team had enough experience to manage the situation without activating the RRS; poor communication and prioritisation by the medical team involved; and failure to repeat abnormal observations. Taken together these reasons suggest that although staff recognised that their patients were in difficulty, they elected not to activate the RRS while further clinical investigations and clinical reviews were pending. Half of respondents felt that the clinical situation was under control in the ward setting. To a certain extent, data from the point prevalence study validate the experience of the bedside clinical staff; namely that among the 23 patients who met RRS activation criteria, only one had a serious adverse event. The other 22 patients had no adverse consequences irrespective of whether the RRS was activated or not. These results suggest that from the perspective of bedside staff there is not sufficient 'face validity' in the sensitivity and specificity of the RRS activation criteria. Therefore bedside staff re-prioritise the requirement for RRS activation based on the particular clinical scenario, taking into account a multitude of 
Table 3 Thematic analysis of structured interview responses from the prospective data collection phase. All patients experienced a missed RRS call with an adverse clinical event

\begin{tabular}{lc}
\hline Characteristics of interviewees & Frequency of responses (n=83), $\mathbf{n}(\%)$ \\
\hline Junior ward nurse & $28(33.7)$ \\
Senior ward nurse & $16(19.3)$ \\
Junior doctor (intern/resident) & $16(19.3)$ \\
Senior doctor (registrar/consultant) & $13(15.7)$ \\
Other (eg ICU outreach nurse) & $18(21.7)$ \\
Actions performed prior to activating RRS & $43(51.8)$ \\
Awaited further review or response by medical staff & $42(50.6)$ \\
Specific treatment or investigations delaying RRS activation & $28(33.7)$ \\
Involved ICU outreach or requested ICU review & $10(12.0)$ \\
Involved senior nursing staff & $45(54.2)$ \\
Explanation as to why RRS was not activated & $25(30.1)$ \\
Felt the situation was under control in the ward setting & $14(16.9)$ \\
ICU team already involved but no ICU bed was available & $13(15.7)$ \\
Team involved were experienced in this type of patient and & $8(9.6)$ \\
felt RRS activation was not required & $6(7.2)$ \\
Poor communication/prioritisation by medical team & $4(4.8)$ \\
Additional skills were not required to manage the patient & $1(1.2)$ \\
No further clinical observations had been taken & \\
Altered thresholds for RRS activation but not documented & \\
Thought they were too junior to activate RRS &
\end{tabular}

factors: the environment, the status of pending investigations, the perceived competence of attending medical staff, and the degree of involvement of critical care or critical outreach. However, one RRS missed call resulted in prolonged critical care admission with multiple reoperations. Potentially, if timely RRS activation had occurred this could have been avoided. Therefore, from an administrative and more specifically critical care viewpoint, prevention of these types of critical admissions is of huge benefit to the patient and the hospital as a whole. However, from the viewpoint of bedside staff, in most instances of RRS activation breach, the patients come to no harm. This may explain why it takes so long for the RRS to be completely accepted within the culture of a particular hospital system, simply because the frequency of useful RRS activations is low and that it takes years for bedside staff to accept and value such a system.

An additional but less common reason for not activating the RRS was prior involvement of critical care teams in the management of patients who fulfilled activation criteria. Thirty per cent of respondents stated that if the critical care team had been involved with the patient (and particularly if no critical care bed was available) then the requirement for a RRS call was negated. This may reflect a perception that critical care teams should manage critically ill patients in the critical care unit and that other critically ill patients in the hospital should be managed by other clinical teams with the assistance of the critical care team.

Across the four hospitals in this study there was a different pattern of events during the study period.
Casey, one of the newer (2002) smaller community hospitals with no on-site critical care service, had 18 RRS activations, none of which were assessed as being missed. The other smaller hospital, Moorabbin, an elective surgical facility that also has no on-site critical care service, had 22 RRS activations but only one missed RRS call. In contrast, the two large acute tertiary referral centres accounted for almost all the missed RRS calls and accounted for all but one of the subsequent interviews. Although both hospitals had a similar RRS activation rate the incidence of missed RRS calls was much less at Dandenong (0.60 vs 1.46 events per 1000 acute adult bed days). As such, the majority of the staff interviews took place at Clayton. Also, at Dandenong, the incidence of cardiac arrest calls and unplanned critical care admissions was less than half that at Clayton. This may reflect a difference in case mix at these two hospitals as Clayton has a number of tertiary specialty referral units (neurology, renal, cardiac and neuro-surgery) that are not present at Dandenong. Alternatively, these differences may be because Dandenong had a RRS in place for 13 years at the time of the study whereas the RRS at Clayton had only been in place for 3 years. Despite this, there were still 13 missed RRS calls across the point prevalence and prospective parts of this study at Dandenong.

\section{Strengths and weaknesses of the study}

The main limitation of this study was the relatively low incidence of missed RRS calls that could be explained by the maturity of the RRS system. Consequently fewer interviews than anticipated could be performed during 
the point prevalence stage. Despite this, we were able to refine the coding of the qualitative responses for the prospective case collection stage. These qualitative data are contaminated in part by the knowledge that the patients involved had suffered an adverse event (cardiac arrest, unplanned ICU admission or death) following a period of physiological instability. Interviews were abandoned if conducted more than $72 \mathrm{~h}$ following the adverse event. This led to a reduced number of interviews, but ensured that the data collected were fresh in the respondents' minds. Although the data from this study come from a single organisation, the four acute hospitals involved each have unique characteristics and consequently we believe the results can be generalised.

\section{CONCLUSION}

There are two important messages from this study. First, the main reason why staff did not follow the RRS activation protocol was not failure of cognition, but rather local sociocultural factors and intra-professional hierarchies in the clinical areas. On this basis, we question the value of efforts to improve RRS effectiveness by making such patients more identifiable (eg, colour-coded observation charts), the push for an increase in the frequency of RRS activation and even the suggestion that RRS protocols become mandatory. Instead, the results suggest that there should be more effort in understanding individual and bedside cultural issues that may be preventing staff from activating the RRS. For example, in this study, referral to or involvement of critical care teams, particularly when the critical care unit has no beds, may confuse the situation for the general bedside ward staff caring for the patient.

Second, implementing systems of care that significantly alter the traditional hierarchical referral model of care, regardless of their potential benefits, takes years to appropriately implement. As mentioned above, we believe this may reflect the lack of 'face validity' that bedside staff may have for the RRS because of the perceived poor sensitivity and specificity of the activation criteria. The decision of whether to activate the RRS was often made by junior staff members who do not have the clinical experience to safely make this decision. While a RRS call gives junior staff members the opportunity to ask for help if they are uncomfortable with the clinical situation, some clearly believed that their colleagues expect them to cope with some situations. This expectation was confirmed by Stewart ${ }^{27}$ in a study across 21 hospitals in the UK which analysed decisionmaking by pre-registration house officers on whether or not to call for senior help. This study found that the decision to call for help is a complex judgement that balanced the desire for clinical autonomy with the understanding of certain consequences to self, senior colleagues and patients. In our study, there was a common belief that the treating team should be able to deal with problems close to their area of specialty. For example, the cardiothoracic surgery team would be able to manage patients with tachy-arrhythmias but may perhaps struggle to deal with patients who had a drop in their Glasgow Coma Score. When deterioration occurred within the area of specialty the junior staff would be more likely to call senior nurses and doctors within the specialty rather than activate the RRS. This context sensitivity of the RRS has not previously been described but appears to be a strong sociocultural influence on whether junior staff activate the RRS.

Author affiliations

${ }^{1}$ Southern Health Quality Unit, Monash Medical Centre Clayton, Clayton, Melbourne, Australia

${ }^{2}$ Academic Board of Peri-operative Medicine, Monash University, Prahran, Melbourne, Australia

${ }^{3}$ Southern Health Simulation and Skills Centre, Monash Medical Centre, East Bentleigh, Melbourne, Australia

${ }^{4}$ University of Tasmania Rural Clinical School, Burnie, Tasmania, Australia

${ }^{5}$ University of Toronto, Department of Surgery, Toronto, Canada

${ }^{6}$ Monash Medical Centre, Clayton, Melbourne, Australia

Contributors All authors contributed to the design, methodology and manuscript preparation.

Funding This study was supported by a grant of $\$ 75000$ from the Victorian Managed Insurance Agency.

Competing interests None.

Provenance and peer review Not commissioned; externally peer reviewed.

\section{REFERENCES}

1. Wilson RM, Runciman WB, Gibberd RW, et al. The quality in Australian health care study. Med J Aust 1995;163:458-71.

2. Wilson RM, Harrison BT, Gibberd RW, et al. An analysis of the causes of adverse events from the quality in Australian health care study. Med J Aust 1999;170:411-15.

3. Buist MD, Jarmolowski E, Burton PR, et al. Recognising clinical instability in hospital patients before cardiac arrest or unplanned admission to Intensive Care. A pilot study in a tertiary-care hospital. Med J Aust 1999;171:22-5.

4. Bellomo R, Goldsmith D, Russell R, et al. Postoperative serious adverse events in a teaching hospital: a prospective study. Med $J$ Aust 2002;176:216-18.

5. Franklin C, Mathew J. Developing strategies to prevent in-hospital cardiac arrest: analyzing responses of physicians and nurses in the hours before the event. Crit Care Med 1994;22:244-7.

6. Smith AF, Wood J. Can some in-hospital cardio-respiratory arrests be prevented? A prospective survey. Resuscitation 1998;37: 133-7.

7. McQuillan P, Pilkington S, Allan A. Confidential enquiry into the quality of care before intensive care unit admission. $B M J$ 1998;316:1853-8.

8. Jones DA, DeVita MA, Bellomo R. Rapid-response teams. N Engl $J$ Med 2011;365:139-46.

9. Chan PS, Jain R, Nallmothu BK, et al. Rapid response teams: a systematic review and meta-analysis. Arch Intern Med 2010;170:18-26.

10. Buist M. The rapid response team paradox: why doesn't anyone call for help? Crit Care Med 2008;36:634-6.

11. Hillman $\mathrm{K}$, Chen $\mathrm{J}$, Cretikos $\mathrm{M}$, et al. Introduction of the medical emergency team (MET) system: a cluster-randomised controlled trial. Lancet 2005;366:2091-7.

12. Chen J, Bellomo R, Flabouris A, et al. The relationship between early emergency team calls and serious adverse events. Crit Care Med 2009;37:148-53. 
13. Santamaria J, Tobin A, Holmes J. Changing cardiac arrest and hospital mortality rates through a medical emergency team takes time and constant review. Crit Care Med 2010;38:445-50.

14. Buist MD, Moore GE, Bernard SA, et al. Effects of a medical emergency team on reduction of incidence of and mortality from unexpected cardiac arrests in hospital: preliminary study. BMJ 2002;324:387-90

15. Buist M, Harrison J, Abaloz E, et al. Six year audit of cardiac arrests and medical emergency team calls in an Australian outer metropolitan teaching hospital. BMJ 2007;335:1210-12.

16. Ciavarella F. Southern Health risk management report 2007-2008. In: Ciavarella F, ed. Southern Health Risk Management Report 2007-2008. Melbourne: Australia, 2008.

17. Jones D, Baldwin I, McIntyre T, et al. Nurses' attitudes to a medical emergency team service in a teaching hospital. Qual Saf Health Care 2006;15:427-32.

18. George AL, Folk BP 3rd, Crecelius PL, et al. Pre-arrest morbidity and other correlates of survival after in-hospital cardiopulmonary arrest. Am J Med 1989;87:28-34.

19. Bedell SE, Deitz DC, Leeman D, et al. Incidence and characteristics of preventable iatrogenic cardiac arrests. JAMA 1991;265:2815-20.
20. Tortolani AJ, Risucci DA, Rosati RJ, et al. In-hospital cardiopulmonary resuscitation: patient and resuscitation factors associated with survival. Resuscitation 1990;20:115-28.

21. Schein RM, Hazdat N, Pena M, et al. Clinical antecedents to in-hospital-cardiopulmonary arrest. Chest 1990;98:1388-92.

22. Marshall SD, Kitto S, Shearer W, et al. Why don't hospital staff activate the Rapid Response System (RRS)? How frequently is it needed and can the process be improved? Implement Sci 2011;6:39.

23. Weigmann DA, Shappell SA. Managing Human Error in Complex Systems. Aldershot, UK: Ashgate, 2008.

24. Endsley MR. Towards a theory of situation awareness in dynamic systems. Hum Factors 1995;37:32-64.

25. Flick U. From text to theory: coding and categorizing. An Introduction to Qualitative Research. 4th edn. London: Sage, 2009:305-32.

26. Harrison J, Buist MD, Flanagan B. A simulation-based MET training course for physician trainees at Southern Health. Abstract Book, The 9th National Forum for Pre-Vocational Medical Education; 24-26 October 2004, Melbourne.

27. Stewart J. To call or not to call: a judgement of risk by pre-registration house officers. Med Educ 2008;42:938-44. 